\title{
A new class of inhomogeneous cosmological models with electromagnetic field in normal gauge for Lyra's manifold.
}

\begin{abstract}
A new class of exact solutions of Einstein's modified field equations in inhomogeneous space-time for perfect fluid distribution with electromagnetic field is obtained in the context of normal gauge for Lyra's manifold. We have obtained solutions by considering the time dependent displacement field. The source of the magnetic field is due to an electric current produced along the z-axis. Only F12 is a non-vanishing component of the electromagnetic field tensor. It has been found that the displacement vector $\beta(t)$ behaves like the cosmological constant $\Lambda$ in the normal gauge treatment and the solutions are consistent with the recent observations of Type Ia supernovae. Physical and geometric aspects of the models are also discussed in the presence of magnetic field.
\end{abstract}

Keyword: Inhomogeneous cosmology; Lyra manifold; Electromagnetic field. 